\title{
DECONVOLUÇÃO PREDITIVA DE REFLEXÕES MÚLTIPLAS E PEG-LEGS UTILIZANDO FILTRAGEM WIENER-LEVINSON MULTICANAL
}

\author{
Adriano P. Lima' \& Milton J. Porsani ${ }^{2}$
}

Received October 25, 1999 / Accepted April 10, 2003

\begin{abstract}
No presente trabalho utilizamos o método de deconvolução preditiva multicanal (DPM) de WienerLevinson (WL) para a supressão de reflexões múltiplas associadas à lâmina d'agua e peg-legs. Os filtros multicanais de WL são obtidos resolvendo-se sistemas de equações normais com estrutura bloco-Toeplitz, formados a partir dos coeficientes da função de auto-correlação e cross-correlação dos traços sísmicos. Tais filtros exploram de forma mais efetiva a redundância espacial e temporal dos sismogramas, comparado aos correspondentes filtros monocanais. A DPM foi aplicada em painéis de traços sísmicos corrigidos de NMO utilizando-se a velocidade da múltipla a ser removida. Neste domínio as múltiplas apresentam caráter periódico e o método multicanal se mostrou bastante eficaz. Exemplos numéricos utilizando dados sintéticos e dados sísmicos marítimos do Golfo do México ilustram o desempenho da filtragem WL multicanal. Os resultados obtidos demonstram que o método de DPM utilizado é robusto e eficaz na remoção de múltiplas e peg-legs.
\end{abstract}

Palavras-chave: Deconvolução sísmica; Filtros multicanais Wiener-Levinson ; Sistemas BlocoToeplitz.

PREDICTIVE DECONVOLUTION OF MULTIPLES AND PEG-LEGS BY USING WIENERLEVINSON MULTICHANNEL FILTERING - In the present paper we have used multichannel predictive filters of Wiener-Levinson (WL) type to deconvolve surface and internal multiples and peg-legs. The WL multichannel filters are obtained as a solution for block-Toeplitz systems of normal equations. These systems are formed with coefficients of auto and cross-correlations of a set of seismic traces. The obtained filters can explore in a more effective way the in-time and spatial redundancy of information that exist in the seismograms.To place the seismic data in a more appropriate way to be deconvolved we have applied the Multiple Moveout (MMO) transform. By forming panels of common-offset the WL multichannel filters were applied. This multichannel predictive deconvolution (MPD) approach was tested using synthetic and marine seismic data. Numerical examples using marine seismic data from the Gulf of Mexico illustrate the performance of the method. The results have been quite satisfactory, thus demonstrating that the method is robust and effective to remove the multiple and peg-leg reflections.

Key words: Seismic deconvolution; Multi-channel Wiener-Levinson's filters; Block-Toeplitz systems.

${ }^{1}$ PETROBRAS S.A. - E\&P-ES/GEXP/GEOF

São Mateus, Espírito Santo, Brazil.

${ }^{2}$ Centro de Pesquisa em Geologia e Geofísica,

Instituto de Geociências, Universidade Federal da Bahia

Campus Universitário da Federação, Salvador, Bahia, Brazil

E-mail: porsani@cpgg.ufba.br 


\section{INTRODUÇÃO}

A deconvolução preditiva (DP) é normalmente empregada no processamento de dados sísmicos com o objetivo de comprimir o pulso sísmico ou predizer e "suprimir" reflexões múltiplas. O primeiro objetivo é alcançado utilizando-se o operador de erro de predição unitária que corresponde ao filtro inverso de Wiener-Levinson (WL). Para a predição e atenuação de reflexões múltiplas utiliza-se o filtro preditivo com a distância de predição pouco menor que o período da múltipla que se deseja suprimir (Robinson, 1984; Robinson \& Treitel, 1980; Yilmaz, 1990). O filtro preditivo de Wiener-Levinson estima eventos periódicos, como múltiplas no sismograma, permitindo assim que a componente estimada seja removida.

Entretanto, nos sismogramas, as múltiplas não se apresentam de forma periódica. A periodicidade inexiste nos traços obtidos para afastamentos não nulos, conforme é o caso de traços de uma família CMP ou de uma família de traços com ponto de tiro comum. Nestes casos, a falta de periodicidade das múltiplas é responsável pela ineficácia da DP.

A transformada Tau-p linear, através da decomposição de dados $x$-t em ondas planas, pode contornar o problema da não periodicidade para dados que seguem um modelo 1-D (Lokshtanov, 1998). No domínio p-comum, mesmo em dados provenientes de áreas com pequenas variações na inclinação do fundo do mar, o problema da não periodicidade das múltiplas poderia ser minorado empregando-se DPM, que iria explorar de forma mais eficiente a redundância de informações.

Um outro domínio de representação dos dados, mais apropriado para o emprego da DPM, no qual as múltiplas são periódicas, pode ser formado por painéis de afastamento-comum de traços que foram corrigidos de NMO com a velocidade de empilhamento da reflexão múltipla que se deseja suprimir. Essa transformada é conhecida na literatura por MMO (Multiple Moveout). Com a aplicação da transformada MMO em dados registrados sobre um fundo do mar horizontal, tanto a reflexão primária quanto as múltiplas do fundo do mar tornam-se horizontais e periódicas com distância de predição igual ao tempo zero-offset da primária. Infelizmente esses eventos não formam uma série estacionária no tempo nem no espaço, devido ao estiramento causado pela correção de moveout. O problema da não estacionariedade das múltiplas no espaço pode ser resolvido através da organização dos traços para o domínio afastamento-comum. Entretanto, para contornar o problema da não estacionariedade no tempo, algo como uma transformada ISR (isostretch-radial) (Lamount et al., 1997) poderia ser empregada.

No presente trabalho utilizamos a DPM para a remoção de múltiplas associadas aos dados sísmicos marítimos do Golfo do México (Lima, 1999). Os filtros multicanais correspondem a operadores bidimensionais que são obtidos como solução de sistemas de equações bloco-Toeplitze implicitamente levam em conta a coerência lateral dos eventos de traços vizinhos (Barros \& Porsani, 1996). Para minorar o problema da não periodicidade das múltiplas, utilizamos a transformada MMO e para explorar as vantagens da DPM trabalhamos com o método sobre painéis de traços de afastamentocomum. Nesse domínio a DPM foi aplicada em cascata e a distância de predição e o número de coeficientes foram determinados em função do período das múltiplas. Os dados assim preparados foram submetidos à DPM.

A seguinte estratégia, foi adotada no presente trabalho:

1) Correção de divergência esférica;

2) Deconvolução do pulso sísmico;

3) Aplicação de MMO direta;

4) Organização dos dados para o domínio afastamento-comum;

5) Aplicação do filtro multicanal;

6) Organização dos dados filtrados para o domínio CMP;

7) Aplicação de MMO inversa.

\section{FILTRAGEM MULTICANAL \\ WIENER-LEVINSON}

Um operador preditivo multicanal age sobre vários traços sísmicos simultaneamente. A teoria multicanal pode ser obtida a partir da monocanal, pela substituição apropriada de escalares por matrizes. 
Considerando-se o caso de dois canais e um filtro de 3 coeficientes em cada canal, podemos escrever,

$$
\tilde{z}_{t}=\sum_{k=1}^{3} x_{t-k+1} a_{k}+\sum_{k=1}^{3} y_{t-k+1} b_{k} .
$$

Ou sob a forma matricial,

$$
\left[\begin{array}{c}
z_{0} \\
z_{1} \\
z_{2} \\
\vdots \\
z_{M} \\
\vdots \\
\vdots
\end{array}\right]=\left[\begin{array}{cccccc}
x_{0} & y_{0} & 0 & 0 & 0 & 0 \\
x_{1} & y_{1} & x_{0} & y_{0} & 0 & 0 \\
x_{2} & y_{2} & x_{1} & y_{1} & x_{0} & y_{0} \\
\vdots & \vdots & \vdots & \vdots & \vdots & \vdots \\
x_{M} & y_{M} & \vdots & \vdots & \vdots & \vdots \\
0 & 0 & x_{M} & y_{M} & \vdots & \vdots \\
0 & 0 & 0 & 0 & x_{M} & y_{M}
\end{array}\right]\left[\begin{array}{c}
a_{1} \\
b_{1} \\
a_{2} \\
b_{2} \\
a_{3} \\
b_{3}
\end{array}\right]
$$

A expressão para os desvios entre valores observados $Z_{\mathrm{j}}$ e calculados $\hat{Z}_{\mathrm{j}}$ pode ser representada na forma matricial como segue:

$$
\mathbf{e}=\mathbf{z}-\mathbf{W}_{0} \mathbf{h}_{0}-\mathbf{W}_{1} \mathbf{h}_{1}-\mathbf{W}_{2} \mathbf{h}_{2}
$$

onde,

$$
\mathbf{h}_{\mathbf{j}}=\left(\begin{array}{ll}
a_{j} & b_{j}
\end{array}\right)^{\mathbf{T}} \cdot \mathbf{W}_{\mathbf{j}}
$$

$W_{i}$ é a matriz formada pelas colunas $j+1$ e $j+2$ da matriz representada pela equação (2). Minimizando a forma quadrática:
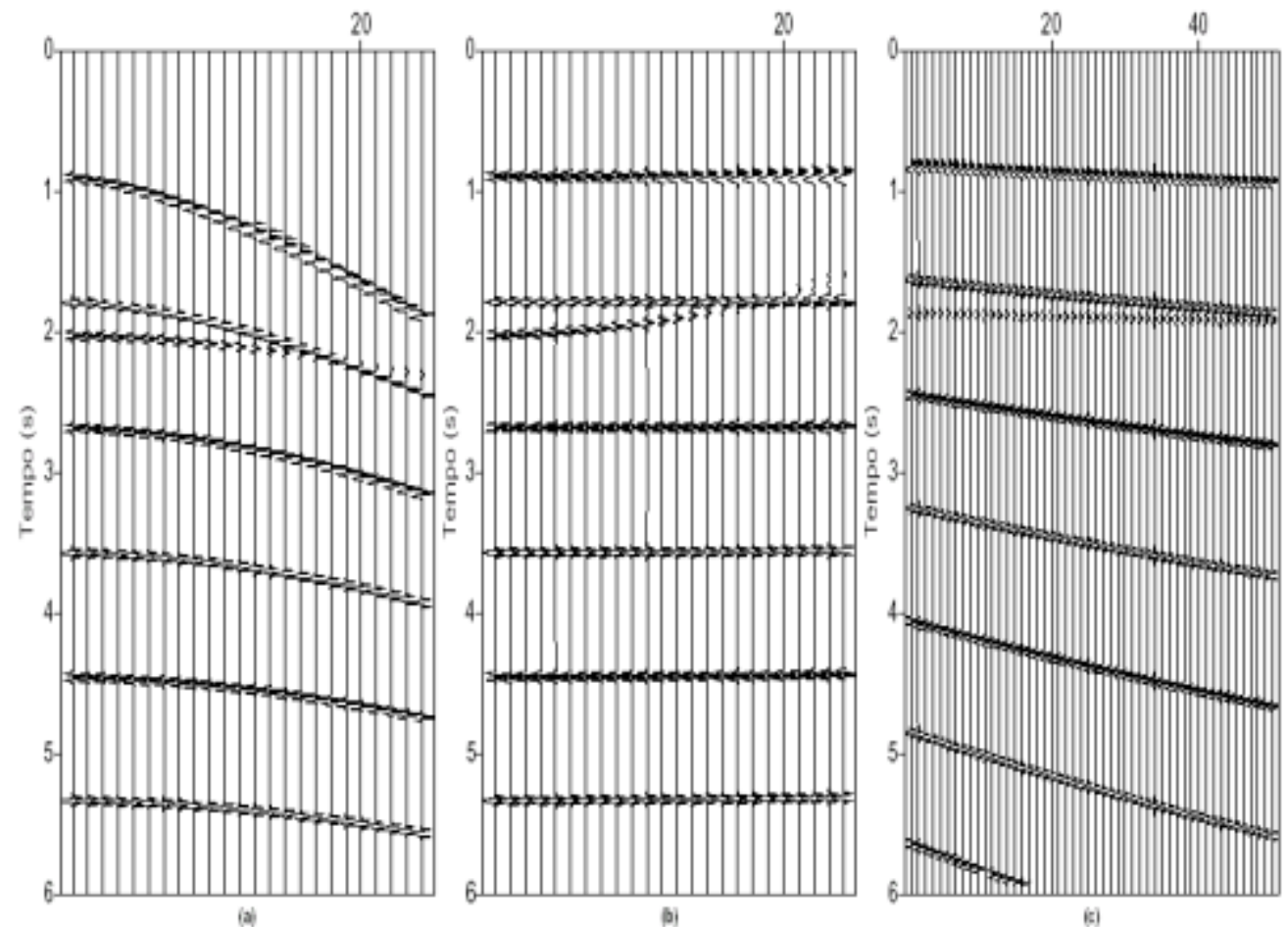

Figura 1 - Efeito da correção de MMO sobre os traços de uma família CMP de dados sintéticos. CMP com reflexões múltiplas em (a). O mesmo CMP após a correção de MMO utilizando-se a velocidade da múltipla, em (b). Painel de afastamento-comum formados por traços corrigidos de MMO mostrando a periodicidade das múltiplas, em (c).

Figure 1 - Effect of MMO correction over a CMP gather of synthetic traces. CMP with multiple reflections in (a). CMP after MMO correction using the velocity associated with the multiple, in (b). Panel of common-offset formed by traces of CMP gathers after MMO correction showing the periodicity of the multiples in $(\boldsymbol{c})$.

$$
Q(\mathbf{h})=\mathrm{e}^{\mathrm{T}} \mathrm{e}
$$

com relação aos parâmetros $\left\{a_{j}, b_{j}\right\}$ obtém-se o sistema de equações normais,

onde:

$$
\left[\begin{array}{ccc}
\mathbf{R}_{0} & \mathbf{R}_{-1} & \mathbf{R}_{-2} \\
\mathbf{R}_{1} & \mathbf{R}_{0} & \mathbf{R}_{-1} \\
\mathbf{R}_{2} & \mathbf{R}_{1} & \mathbf{R}_{0}
\end{array}\right]\left[\begin{array}{l}
\mathbf{h}_{0} \\
\mathbf{h}_{1} \\
\mathbf{h}_{2}
\end{array}\right]=\left[\begin{array}{l}
\mathbf{W}_{0}^{T} \mathbf{z} \\
\mathbf{W}_{1}^{T} \mathbf{z} \\
\mathbf{W}_{2}^{T} \mathbf{z}
\end{array}\right]
$$

$$
\begin{array}{ll}
\mathbf{R}_{0}=\mathbf{W}_{\mathbf{j}}^{\mathbf{T}} \mathbf{W}_{\mathbf{j}} & j=0,1,2 \\
\mathbf{R}_{1}=\mathbf{W}_{\mathbf{j}}^{\mathbf{T}} \mathbf{W}_{\mathbf{j}+1} & j=0,1 \\
\mathbf{R}_{2}=\mathbf{W}_{\mathbf{j}}^{\mathbf{T}} \mathbf{W}_{\mathbf{j}+2} & j=0 \\
\mathbf{R}_{-\mathbf{j}}=\mathbf{R}_{\mathbf{j}}^{\mathrm{T}} & j=0,1,2
\end{array}
$$

A matriz dos coeficientes na eq. (4) possui estrutura bandeada Toeplitz formada por blocos de matrizes. A solução do sistema pode ser obtida com a recursão de Levinson multicanal (Porsani, 1993). Ao invés de escalares, temos blocos de matrizes quadradas de dimensões $n_{\mathrm{c}} \times n_{\mathrm{c}}$ onde $n_{\mathrm{c}}$ representa $\mathrm{o}$ número de canais que correspondem aos traços sísmicos escolhidos para a geração do filtro.

Deixando o sinal desejado corresponder a um dos canais de entrada, avançado de $\mathrm{L}$ amostras, $\mathrm{z}_{\mathrm{t}}=$ 

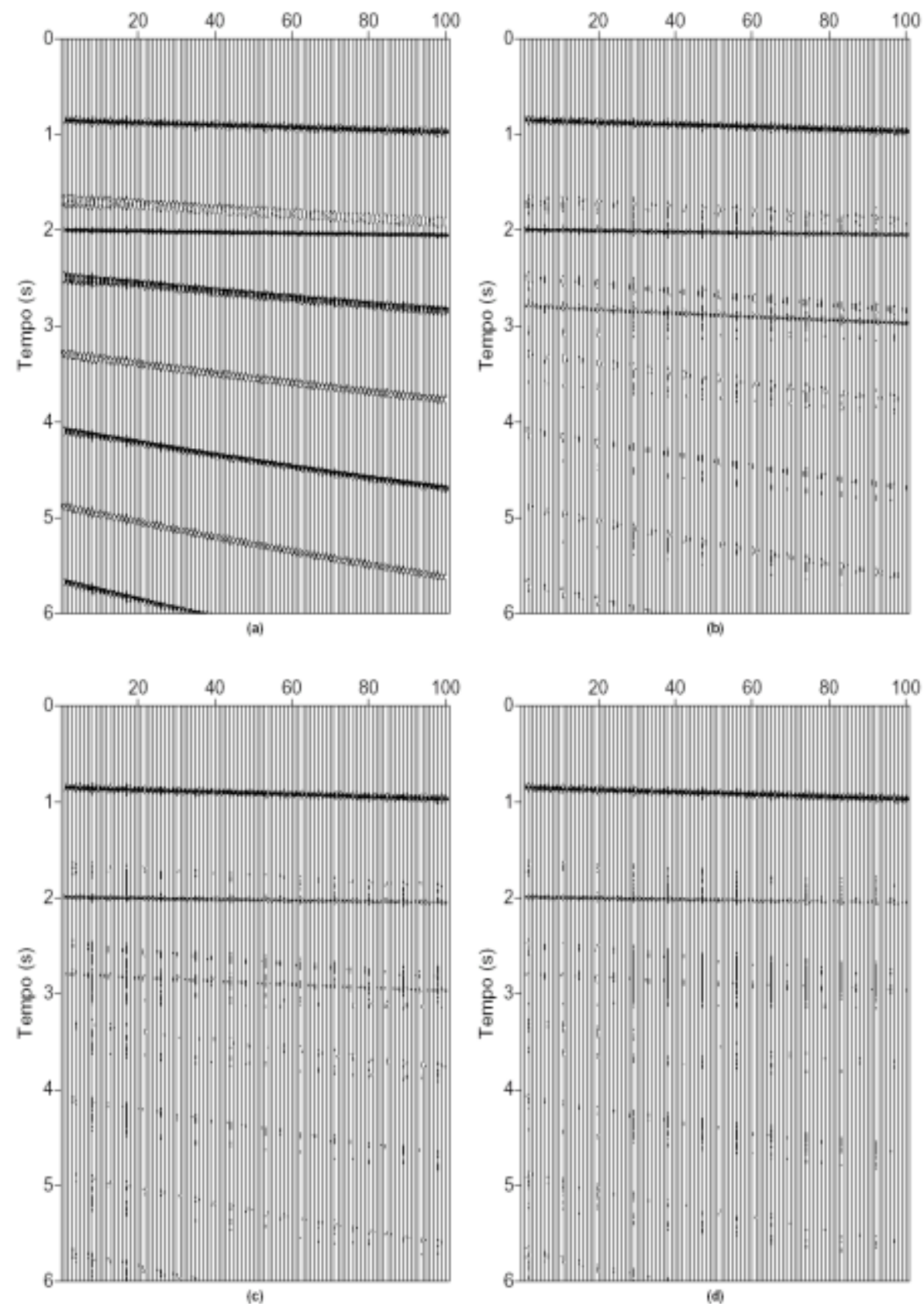

Figura 2 - Seções empilhadas dos dados sintéticos mostrando resultados da aplicação da DPM no domínio do afastamento-comum. Seção empilhada sem DP em (a). Seção empilhada após DP monocanal em (b). Seções empilhadas após DPM com 3 canais em (c) e com 5 canais em (d).

Figure 2 - Stacked sections of synthetic data showing results of the MPD method applied in the common-offset domain (a). Stacked section after monochannel predictive deconvolution in (b). Stacked sections after MPD using 3 channels in (c) and using 5 channels in (d).

$\mathrm{x}_{\mathrm{t}+\mathrm{L}}$, teremos, na solução da eq. (4), os coeficientes do operador preditivo multicanal com distância de predição L. Se o número de canais for apenas um, o método reduz-se ao métodoconvencional de WienerLevinson para DP monocanal. $\mathrm{Se} \mathrm{L}=1$ teremos os coeficientes do operador de predição unitária, relacionado com o filtro inverso de WL utilizado na deconvolução do pulso sísmico.

\section{APLICAÇÃO A DADOS SINTÉTICOS}

Para verificar a aplicabilidade do método de DPM no domínio do afastamento-comum de acordo com a estratégia apresentada, utilizou-se de 100 CMPs de 24 traços e tempo de registro de 6s. Esses dados sintéticos foram gerado com o auxílio do pacote de programas SU (Cohen \& Stockwell,1997), 

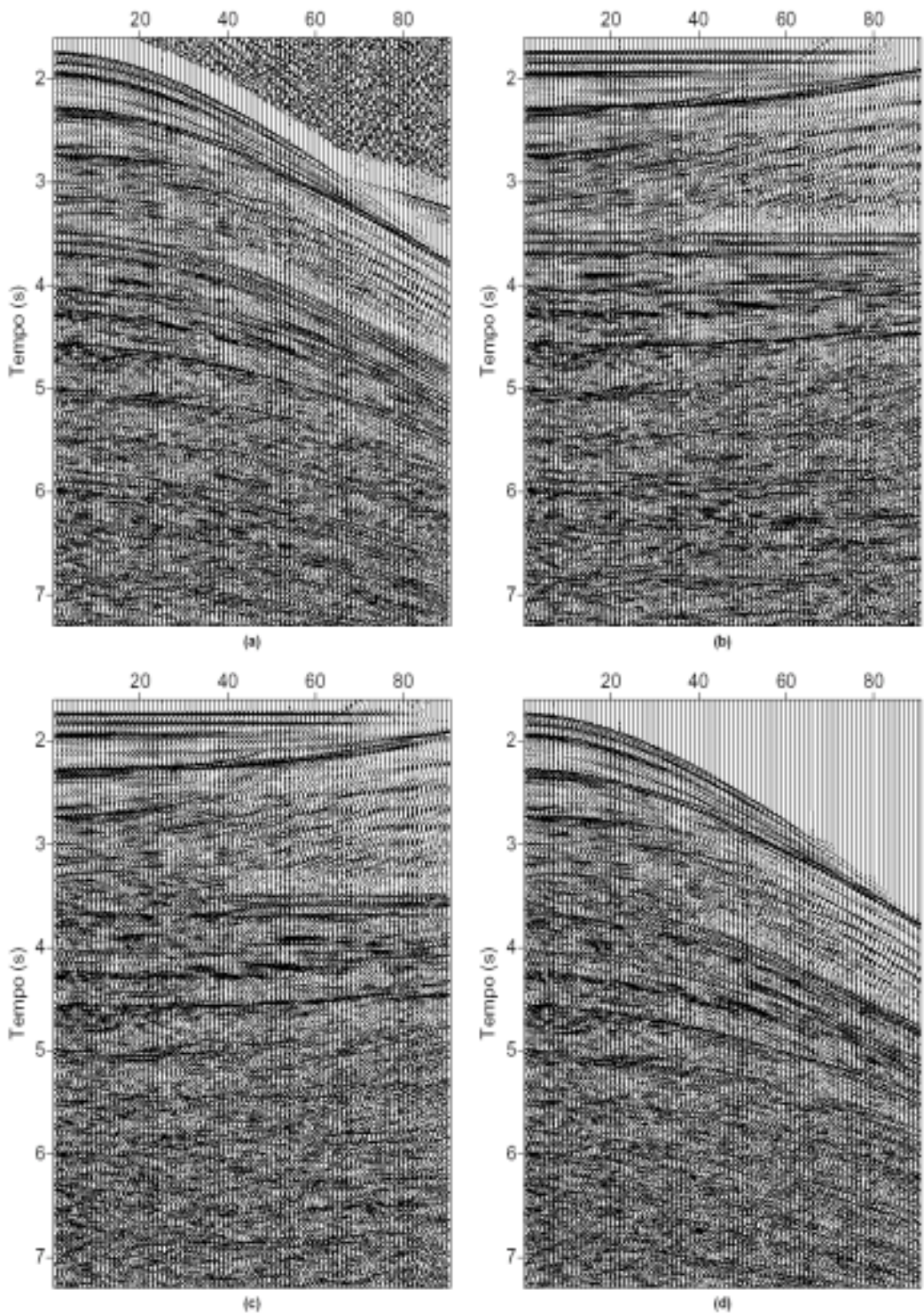

Figura 3 - DPM de um CMP dos dados do Golfo do México. CMP original em (a). CMP após a correção de MMO em (b). CMP reorganizada após a DP no domínio do afastamento-comum para a remoção da múltipla do fundo do mar, ocorrendo em torno de 3,5s, em (c). CMP após a correção inversa de MMO em (d).

Figure 3: Results of application of MPD to a CMP gather from the Gulf of Mexico. Original CMP in (a). CMP after MMO correction in (b). Reorganized CMP after applying MPD in the common-offset domain to deconvolve the multiple associated with the bottom of the sea, visible around $3.5 s$, in (c). CMP after applying the inverse MMO correction in (d).

contendo além da primária e múltiplas correspondendo ao fundo do mar (interface 1), uma outra primária correspondendo a uma segunda interface, de maneira que se podesse analisar o problema da falta de estacionariedade no tempo resultante da correção de MMO.

A Fig. 1 ilustra em (b) a falta de estacionariedade no espaço, a qual é contornada em (c) com a organização dos dados em afastamento-comum. Pode-se verificar em (b) a falta de estacionariedade no tempo nos afastamentos longos, principalmente quando se compara a wavelet da primária da interface $1 \mathrm{com}$ as wavelets correspondentes das múltiplas. Isto deve-se ao maior estiramento da primária em relação as múltiplas, o que foi bastante amenizado quando utilizou-se dados gerados a partir de modelos com lâmina d'água bastante espessa.

A Fig. 2 apresenta as seções estaqueadas com os dados sintéticos. Foram aplicadas as DPs com 1 , 3 e 5 canais utilizando-se uma distância de predição de $750 \mathrm{~ms}$ e comprimento do operador de $250 \mathrm{~ms}$. Pode-se observar um melhor desempenho das DPs multicanais, muito embora a utilização de um maior número decanais afetou a amplitude da primária da 
interface 2. Este problema decorre do fato de que, na região correspondente a um mar mais profundo, essa primária coincide com a múltipla de primeira ordem da interface 1. Esse problema não é resolvido pela transformação de MMO e o filtro multicanal, apesar de atuar melhor que o monocanal, atua também sobre a primária reduzindo sua amplitude.

\section{APLICAÇÃO AOS DADOS DO GOLFO DO MÉXICO}

Os dados do levantamento sísmico 2-D do Golfo do México foram adquiridos em mar profundo (cerca de 1500m) com os seguintes parâmetros:

- Número de PTs - 1000

- Número de traços por PT - 180

- Intervalo de PTs - 26,67 m

- Intervalo de receptor $-26,67 \mathrm{~m}$

- Afastamento curto - 100,58 m

- Afastamento longo-4874,66 m

- Tempo de registro $-9,016 \mathrm{~s}$

- Razão de amostragem - 4 ms

As múltiplas de primeira e segunda ordem relativas ao fundo do mar são facilmente identificadas devido ao seu grande período (em torno de 1,8s). A presença de um corpo de sal subjacente ao assoalho oceânico é responsável pela presença de múltiplas de forte amplitude associadas ao topo e base do sal e peg-legs. Tais múltiplas interferem com as reflexões primárias causando sérias dificuldades de imageamento.

Por razões de economia no tempo de processamento e limitações de espaço em disco (1.618Gb para cada conjunto de dados deconvolvido), escolheu-se trabalhar apenas com os CMPs ímpares e com cobertura nominal, resultando 912 CMPs com 90 traços cada.

\section{REMOÇÃO DE MÚLTIPLAS DO FUNDO DO MAR}

Para deconvolver as múltiplas do fundo do mar, utilizamos a estratégia citada anteriormente, efetuando uma correção de MMO com velocidade $1500 \mathrm{~m} / \mathrm{s}$ e aplicando a DPM no domínio afastamento-comum. A distância de predição variou dentro de cada painel de acordo com o tempo da primária do fundo do mar, sendo a mesma para todos os painéis. $\mathrm{O}$ comprimento do operador também variou dentro de cada painel (20\% do tempo da primária do fundo do mar), o número de canais utilizados foi igual a $5 \mathrm{e}$ uma luz branca de $0,3 \%$ foi necessária para evitar a instabilidade numérica do algoritmo quando da computação dos filtros multicanais.

Todos os dados são aqui representados a partir de 1,6s. O CMP original está representado em (a). A Fig. 3b ilustra através de um CMP os resultados da DPM nos dados corrigidos de MMO e reorganizados para o domínio do afastamento-comum. O resultado da correção de MMO está apresentado em (b). Observe a presença da múltipla de primeira ordem do fundo do mar horizontalizada em torno de 3,5s. Em (c) pode-se ver os dados do CMP restituidos dos painéis de afastamento-comum após a DPM. Observe a significativa redução da múltipla do fundo do mar, principalmente nos afastamentos mais curtos. Em (d) tem-se o CMP dos dados mostrados em (c) após a transformada inversa de MMO.

A partir dos dados representados na Fig. 3d foi feito um empilhamento de modo a se poder verificar a eficiência da DPM na atenuação de múltiplas do fundo do mar nos dados do Golfo do México.

A Fig. 4 mostra a seção estaqueada sem DP em (a). A seção empilhada após DPM está representada em (b). Observe a quase total eliminação das reflexões múltiplas do fundo do mar que, além da DPM, foi também beneficiada com um mute na região de maiores estiramentos após o NMO, bem como, com a atenuação propiciada pelo empilhamento das famílias CMPs. Pode-se verificar ainda que a DPM também atingiu a múltipla do topo do sal, em torno de $4 \mathrm{~s}$, indicando que o comprimento do operador utilizado, igual a $20 \%$ da distância de predição, poderia ter sido menor, uma vez que as outras múltiplas serão deconvolvidas posteriormente com diferentes parâmetros.

\section{REMOÇÃO DE MÚLTIPLAS INTERNAS E PEG-LEGS}

A supressão das outras múltiplas (topo e base do sal e peg-legs) existentes nos dados poderia ser parcialmente realizada se aumentássemos o 


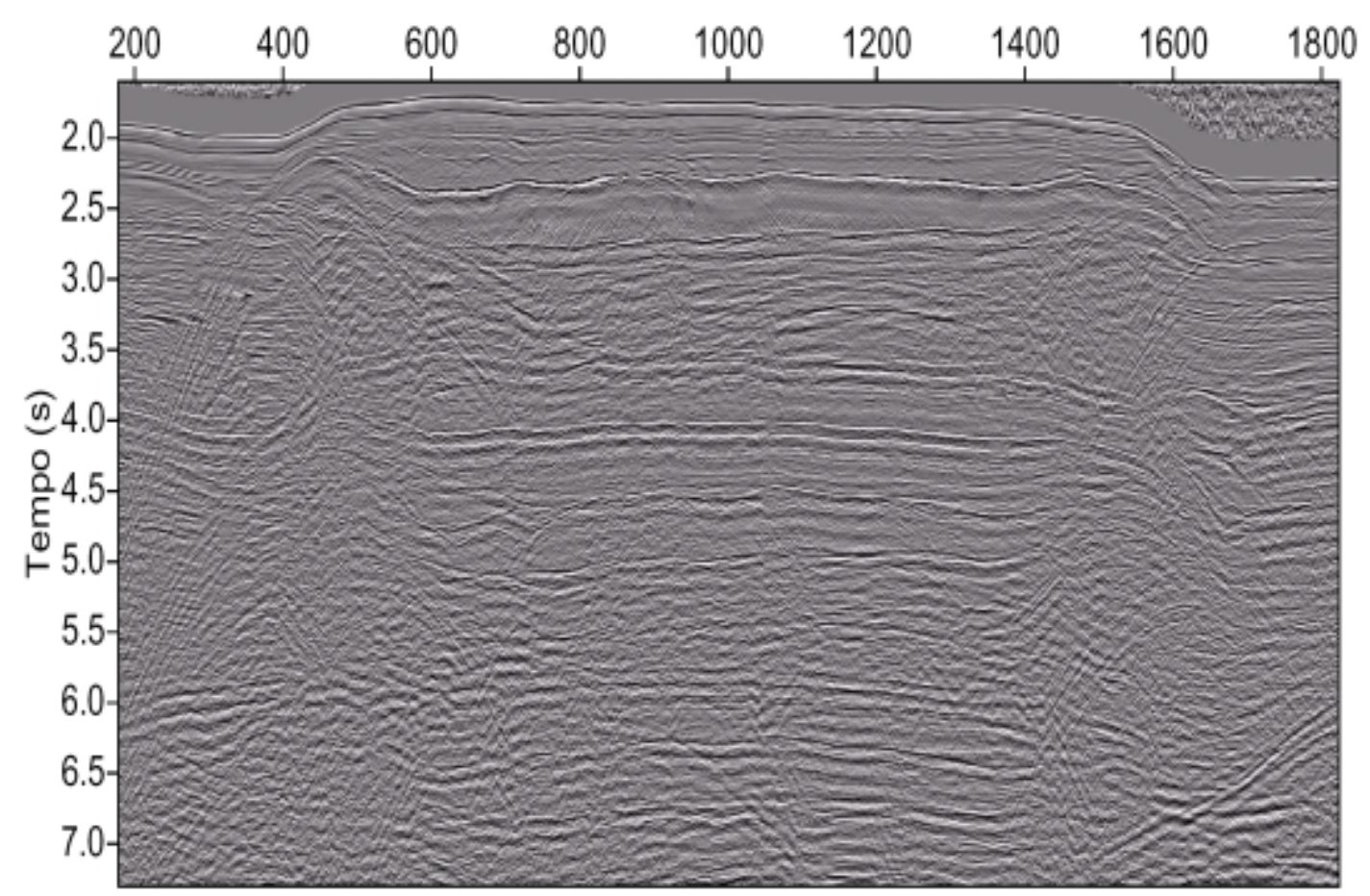

Figura 4 - Seção empilhada dos dados do Golfo do México sem DPM.

Figure 4 - Stacked section of the Gulf of Mexico data without MPD.

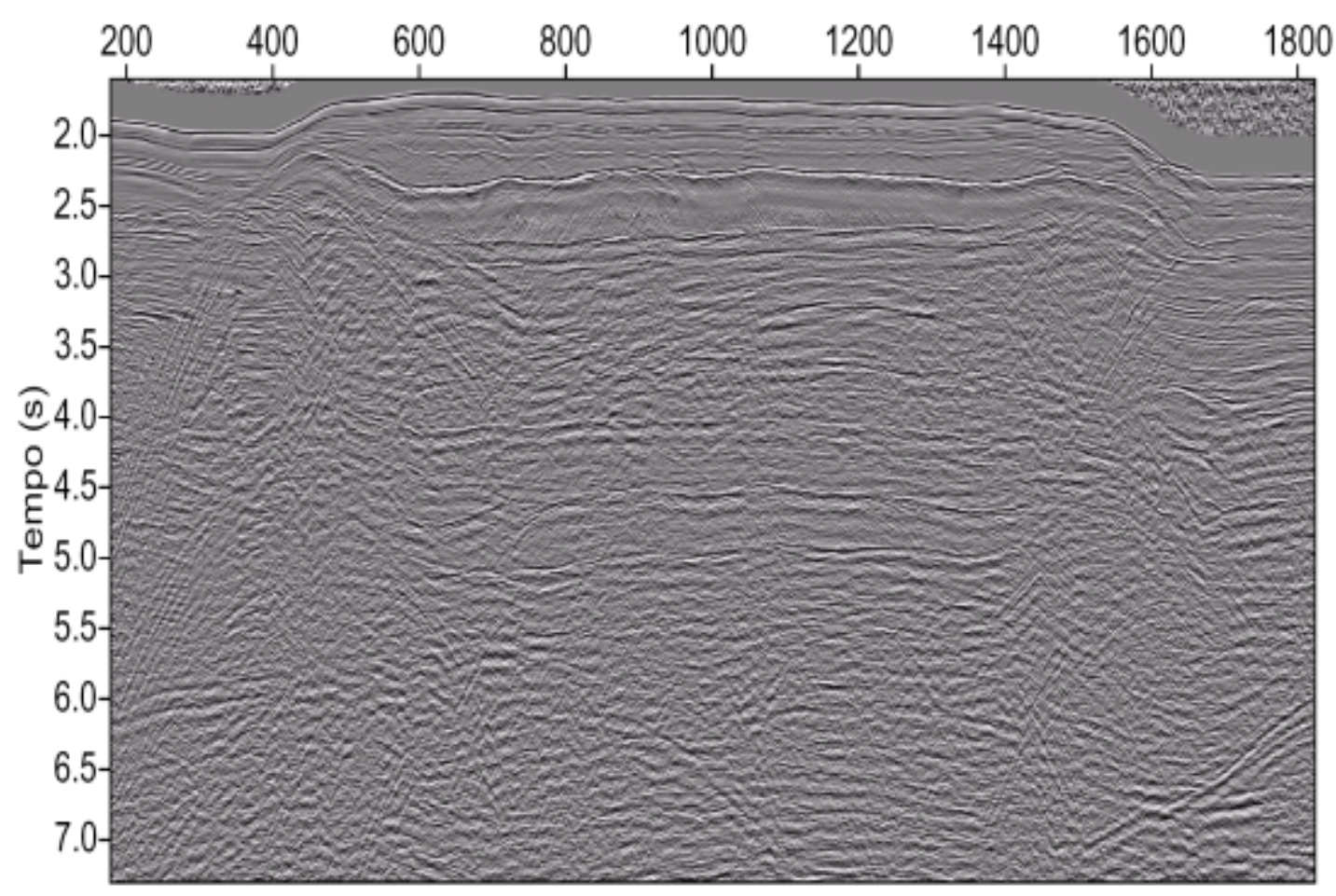

Figura 5 - Seção empilhada dos dados do Golfo do México após a DPM para a remoção da múltipla do fundo do mar, em torno de 3,5s.

Figure 5 - Stacked section of the Gulf of Mexico data after applying the MPD method to remove the multiple associated to the bottom of the ocean, around $3.5 \mathrm{~s}$. 


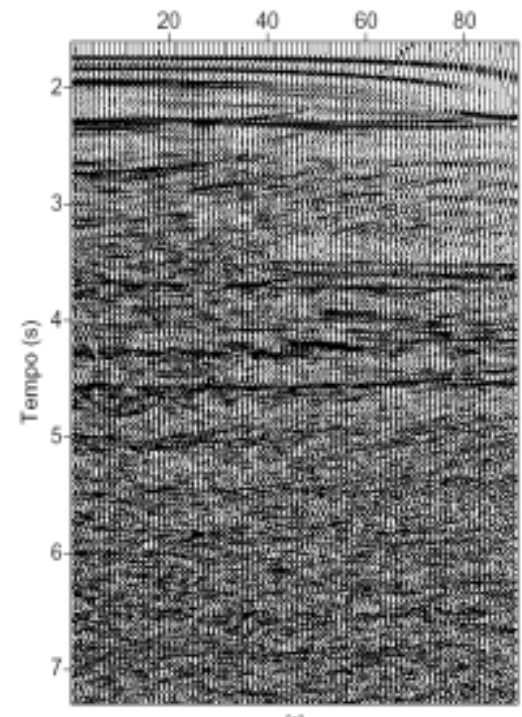

(a)

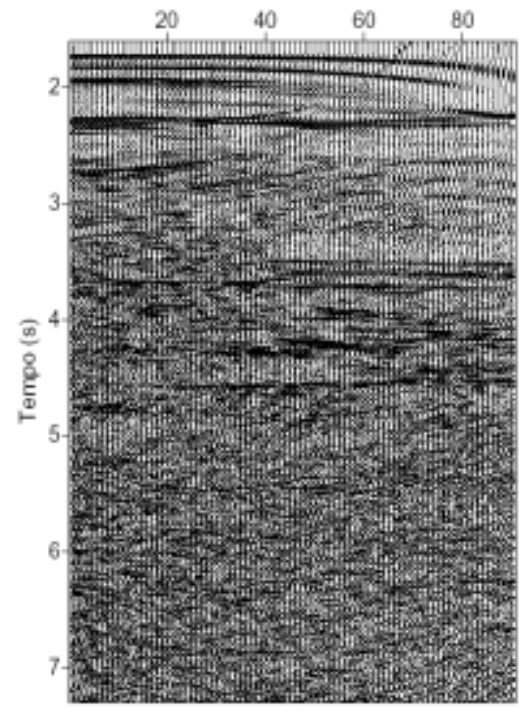

(c)
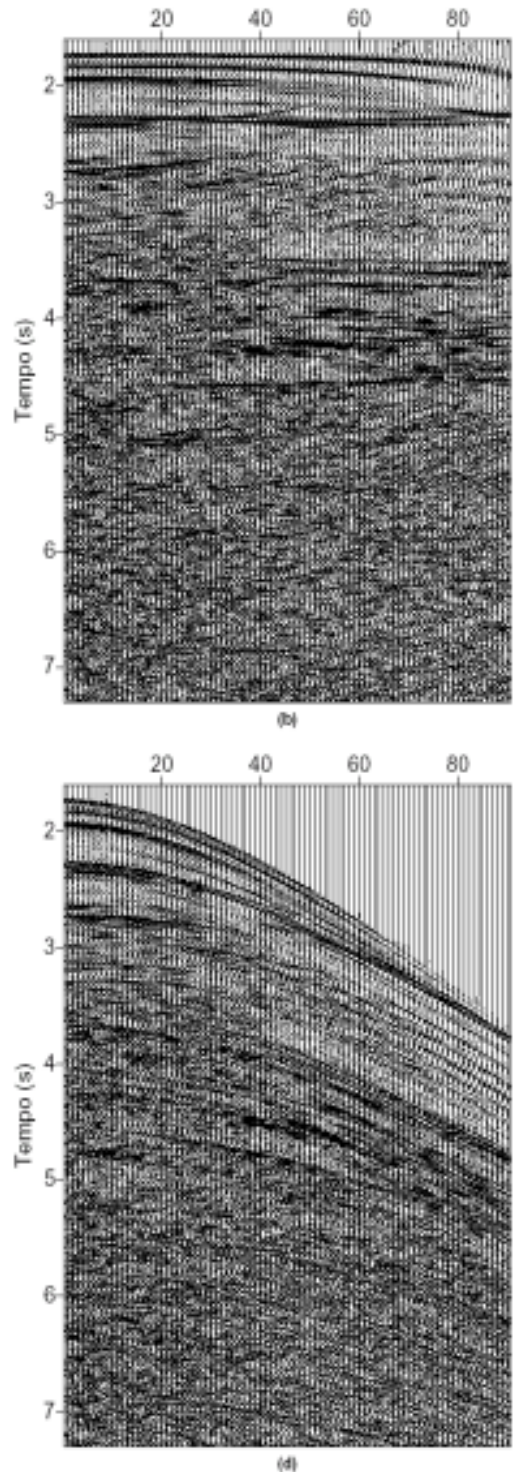

Figura 6 - DPM aplicada em cascata em um CMP dos dados do Golfo do México. CMP após a aplicação de MMO com a velocidade do peg-leg (passo 1) em (a). CMP reorganizado após a DPM em cascata no domínio do afastamento-comum para a atenuação das múltiplas referentes ao topo e base do sal (passo 3) em (b). CMP recomposto após a DPM em cascata para a atenuação da peg-leg (passo 4 e 5) em (c). O mesmo CMP após a correção inversa de MMO (passo 6), em (d).

Figure 6 - Results obtained by sequentially reapplying of the MPD method to a CMP of the Gulf of Mexico. CMP after MMO using the velocity of the peg-leg multiple (step 1) in (a). CMP reorganized after the MPD applied in the common-offset domain to suppress the multiple associated with the top and base of the salt (step 3), in (b). CMP restored after the MPD sequentially applied to suppress the peg-leg (step 4 and 5) in (c). The same CMP after the inverse MMO correction (step 6) in (d).

comprimento do operador, de maneira que a soma da distância de predição e comprimento do operador fosse maior que duas vezes o período das múltiplas (Robinson, 1984). Porém, como a DP utiliza o critério da mínima energia da saída do operador, a distorção nas primárias seria inevitável.

Para a remoção das múltiplas internas a estratégia adotada foi trabalhar em cascata utilizando distâncias de predição e comprimento do operador preditivo adequado a cada múltipla que se pretenda remover.
Para tanto utilizamos os dados com as múltiplas do fundo do mar deconvolvidas e, após a correção inversa de MMO, fizemos uma análise de velocidade para determinar quais funções velocidades melhor horizontalizam as outras múltiplas. Analisou-se também quais distâncias de predição e número de coeficientes do filtro preditivo seriam melhor adequados a cada tipo de múltipla.

Portanto, de acordo com o exposto acima e considerando que agora o dado de entrada já está 


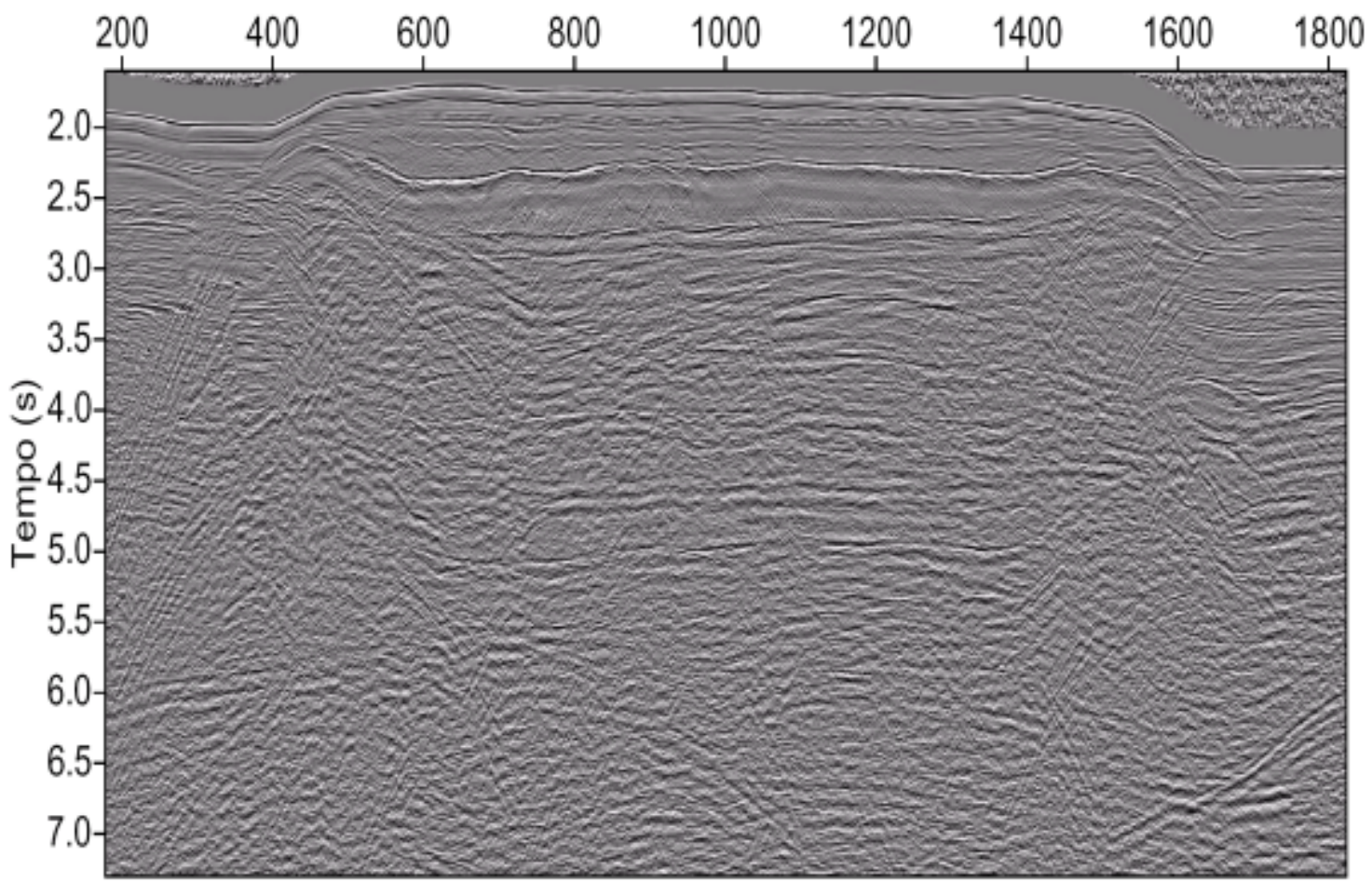

Figura 7 - Seção empilhada dos dados do Golfo do México após a aplicação do passo 3.

Figure 7 - Stacked section of the Gulf of Mexico data after applying step 3.

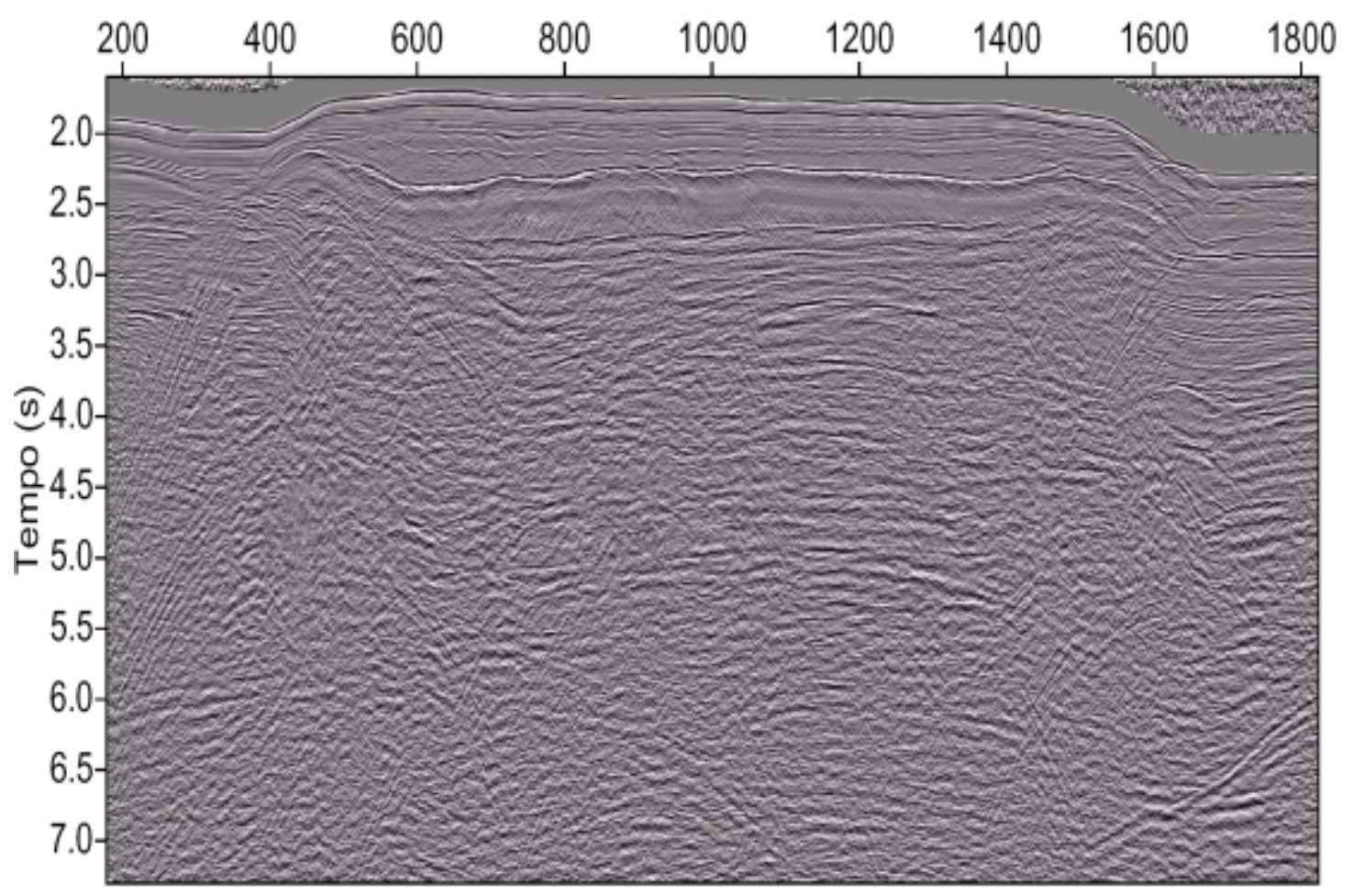

Figura. 8 - Seção empilhada dos dados do Golfo do México após todas as DPs multicanais aplicadas em cascata.

Figure. 8 - Stacked section of the Gulf of Mexico data after sequentially applying all MPDs. 
com a múltipla do fundo do mar atenuada (Fig. 3d), aplicamos os seguintes passos para a atenuação das outras múltiplas:

1) Aplicação de $M M O$ direta com uma função velocidade para tentar horizontalizar as múltiplas do topo e base do sal e peg-leg.

2) Organização dos dados para o domínio do afastamento-comum.

3) Aplicação do filtro multicanal com distância de predição igual a 2,10s e comprimento do operador de 0,30 s.

4) Aplicação do filtro multicanal com distância de predição igual a 2,55s e comprimento do operador de $0,25 \mathrm{~s}$.

5) Organização dos dados filtrados para o domínio CMP.

6) Aplicação de MMO inversa.

7) Proceder com os próximos passos do processamento.

A Fig. 6a mostra uma CMP onde foi feita a nova correção de MMO citada no passo 1. Observa-se que a primária e as múltiplas correspondentes ao sal, que estavam sobrecorrigidas, Fig. 3b, agora encontram-se horizontalizadas. Reorganizando-se os dados para o domínio do afastamento-comum (passo 2) e reaplicando-se a DPM $(\mathrm{NC}=5)$ com distância de predição 2,10 s e comprimento do filtro igual a 0,30 s, e recompondo os traços correspondentes ao CMP obtém-se o resultado apresentado na Fig. 6 b. A seguir aplicou-se em cascata uma outra DPM $(\mathrm{NC}=5)$ com distância de predição $2,55 \mathrm{~s}$ e comprimento do filtro de $0,25 \mathrm{~s}$, conforme o passo 3 . O resultado pode ser visto na Fig. 6c e 6d, que mostra o mesmo CMP da Fig. 3a onde foram aplicadas a DPM para as múltiplas do fundo do mar e mais as duas deconvoluções em cascatas citadas acima. Em $6 c$ e $6 \mathrm{~d}$ pode-se verificar também a significativa atenuação da energia em torno de $5,00 \mathrm{~s}$, referente ao peg-leg, obtida após a aplicação do passo 4.

A Fig. 7 mostra a seção empilhada após o passo 3 , onde podemos observar a atenuação das múltiplas referentes ao topo e base do sal. A Fig. 8 mostra a seção empilhada após a aplicação das três deconvoluções multicanais em cascata. Observar a significativa atenuação das multiplas relativas ao fundo do mar, topo e base do sal, bem como das peg-legs. Comparando-se a seção empilhada original (Fig. 5) com a Fig. 8 podemos observar o excelente desempenho do método de DPM na remoção da múltipla associada ao fundo do mar bem como na remoção das múltiplas internas.

\section{CONCLUSÕES}

Neste trabalho apresentamos o método para obtenção de filtros preditivos multicanais do tipo Wiener-Levinson e o aplicamos à deconvolução de reflexões múltiplas do fundo do mar e peg-legs. Os dados foram processados no domínio afastamentocomum corrigido de MMO e a DPM foi aplicada reiterando-se sobre os dados filtrados com novos parâmetros de filtragem (filtragem em cascata) para remover múltiplas distintas com diferentes períodos de reverberação. Conforme ilustram as figuras, os resultados obtidos são bastante satisfatórios, mostrando que a DPM é mais eficaz que a DP monocanal particularmente nos afastamentos mais curtos. Os resultados indicam que resultados ainda melhores possam ser obtidos no processamento de seções de curtos afastamentos, em dados marítimos de água profunda, vez que o estiramento introduzido pelo MMO supostamente deva ser menor. Na forma como foi utilizado o método de DPMé $n c$ vezes mais dispendioso, ( $n c=$ número de canais), em tempo de processamento, que a DP monocanal.

\section{AGRADECIMENTOS}

Os autores desejam expressar agradecimentos ao geofísico André Romanelli pelas sugestões apresentadas. Milton Porsani agradece ao $\mathrm{CNPq}$ pelo suporte dado à pesquisa e Adriano Lima agradece à PETROBRAS S/A pela oportunidade de realizar o mestrado.

\section{REFERÊNCIAS}

Alan, A. \& Austin, J., 1981. Multiple Suppression using Slant Stacks, Tech. Report, Western Geophisycal Company.

Barros, A. Z. N. \& Porsani, M.J., 1996. Deconvolução de Múltiplas de Longo Período Usando Filtragem Multicanal, Anais Seminário Interno da PETROBRAS. 
Cohen, J. \& Stockwell, J. J. W., 1997. CWP/SU: Seismic Unix Release 30, Center of Wave Phenomena, Colorado School of Mines, Colorado.

Lamont, M. G., Hartley, B.M. \& Ureu, N.F., 1998. 3D Multiple Moveout Wavefield Transformation for Pre-conditionanting data for Removal of Water Bottom Multiple. 68th Ann. Internat. Mtg., Soc. Expl. Geophys., Expanded Abstract Number SP 6.5.

Lima, A. P., 1999. Deconvolução preditiva nos domínios t-x e tau-p utilizando filtros WienerLevinson multicanais, Dissertação (Mestrado), Geofísica, UFBA - Universidade Federal da Bahia.
Lokshtanov, D. E., 1998. Multiple Suppression by single channel and multichannel deconvolution in the tau-p domain. $68^{\text {th }}$ Ann. Internat. Mtg., Soc. Expl.Geophys., Expanded Abstract Number SP 6.4.

Porsani, M. J., 1993. Algoritmo recursivo para solução de sistemas de equações Bloco-Toeplitz. Publicação interna CPGG/UFBA.

Robinson, E. A., 1984. Seismic Inversion \& Deconvolution, Part A: Classical Methods, Geophysical Press.

Robinson, E. A. \& Treitel, S., 1980. Geophysical Signal Analysis, Englewood Gliffs, Prentice-hall, N.J. 466p.

Yilmaz, O., 1990. Seismic Processing, SEG.

\section{PREDICTIVE DECONVOLUTIONOF MULTIPLES AND PEG-LEGS USING WIENER-LEVINSON MULTICHANNELFILTERING}

Introduction: In the present paper we have used multichannel predictive filters of Wiener-Levinson (WL) type to deconvolve surface and internal multiples and peg-legs. The WL multichannel filters are obtained as a solution for block-Toeplitz systems of normal equations (NEs). These systems are formed by coefficients of auto and cross-correlations of a set of seismic traces. To place the seismic data in a more appropriate way to be deconvolved we have applied the Multiple Moveout (MMO) transform.

Wiener-Levinson multichannel filtering: A predictive multichannel filtering acts on many traces simultaneously. The WL multichannel predictive filter may be obtained by solving a block-Toeplitz system of NEs (see eq. 4). The NEs may be solved by using the Levinson's recursion. The obtained filters can explore in a more effective way the in-time and spatial redundancy of information that exist in the seismograms. By forming panels of common-offset the WL multichannel filters were applied. This multichannel predictive deconvolution (MPD) approach was tested using synthetic and marine seismic data.

Numerical examples: Figs. 1a-1c show the effect of MMO correction over a CMP gather of synthetic traces. A proper domain to apply MPD is obtained by forming panels of common-offset traces after MMO correction, where the periodicity of the multiples is better represented. Figs. 2a-2d show stacked sections of synthetic data showing results of the MPD method applied in the common-offset domain. Example of MPD using a CMP gather from the Gulf of Mexico is shown in Figs. 3a-3d. Fig. 4 shows the stacked sections of the Gulf of Mexico data without MPD. Fig. 5 shows the stacked section of the Gulf of Mexico data after applying the MPD method to remove the multiple associated to the bottom of the ocean, around 3.5s. Figs. 6a-6d show results obtained by sequentially reapplying the MPD method to a CMP of the Gulf of Mexico. Fig. 7 shows the stacked section after applying MPD in the common-offset domain to suppress the multiple associated with the top and base of the salt. Fig. 8 shows the stacked section of the Gulf of Mexico data after sequentially applying all MPDs.

Conclusions: Numerical examples using marine seismic data from the Gulf of Mexico illustrate the performance of the MPD method. The results have been quite satisfactory, thus demonstrating that the method is robust and effective to remove the multiple and peg-leg reflections. 


$$
\begin{aligned}
& \mathcal{T H E} 21-35^{\text {th }} \mathcal{M O S} \mathcal{T} \text { VIS I T ED RBG } \mathcal{A R T} \text { I CLES AT } \\
& \mathcal{W} \mathcal{W} \text { W.S CIELO .BR } / \mathcal{R B} \\
& \text { (from Fe 6ruary 26, } 1998 \text { to } g \text { uly 14, 2003) }
\end{aligned}
$$

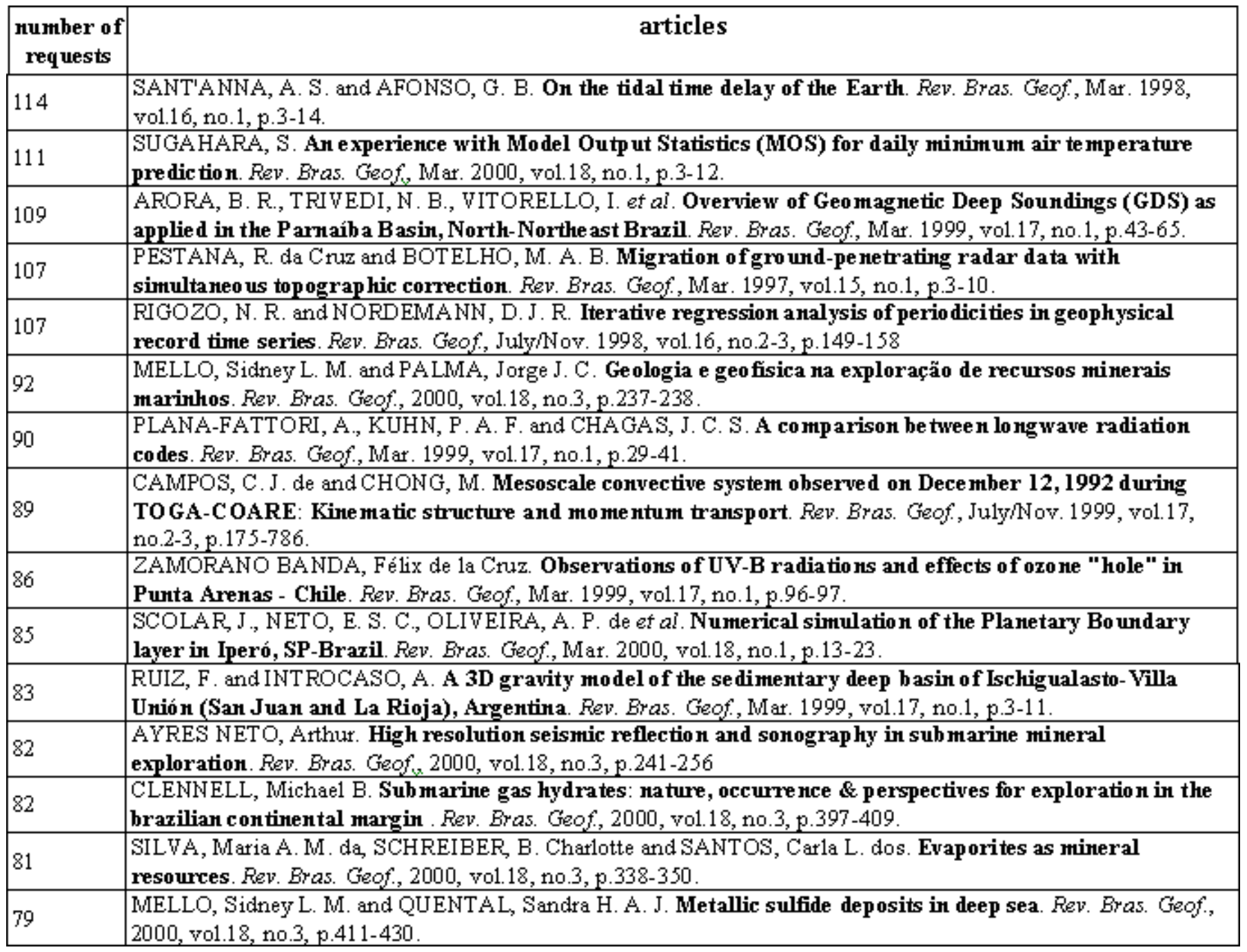

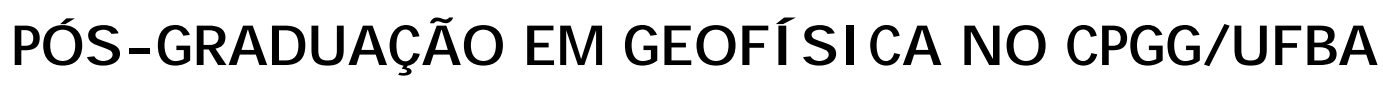

As linfas de pesquisas atualmente em execução no CPGG/UFBA e que dão suporte ao curso de

Pós-graduação e m Ge ofísica são:

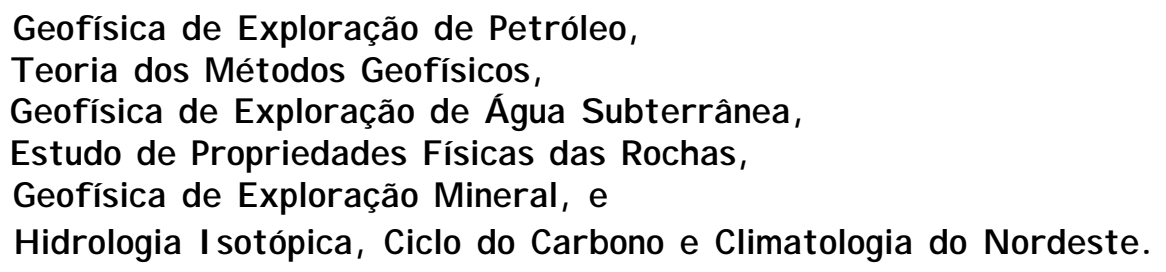

